\title{
COMUNICACIÓN
}

\section{Presencia de Amblyopinodes gahani gahani (Fauvel, 1901) (Coleoptera: Staphylinidae: Ambliopininae) en localidades ribereñas de la Argentina}

\author{
MARCELA LARESCHI****, SANTIAGO NAVA* y ARMANDO C. CICHINO*******
}

\section{PRESENCE OF Amblyopinodes gahani gahani (Fauvel, 1901) (COLEOPTERA: STAPHYLINIDAE: AMBLIOPININAE) IN RIVERINE LOCALITIES FROM ARGENTINA}

In the present study the presence of Amblyopinodes gahani gahani (Fauvel, 1901) in riverine localities from Buenos Aires Province in Argentina, is analyzed. Among six species of rodents captured, A. g. gahani was collected only on Oxymycterus rufus (Fisher, 1814) from Hudson, Punta Lara and La Balandra, and on Scapteromys aquaticus Thomas 1920 from the above mentioned localities, as well as from Los Talas. In the remaining localities, Ramallo and Palo Blanco, where O. rufus has not been captured, nor specimens of $\boldsymbol{A}$. g. gahani have been collected. The results obtained confirm previous knowledgements concerning the relationship between $\boldsymbol{A}$. g. gahani and $\boldsymbol{O}$. rufus.

Key words: Amblyopinodes gahani gahani, beetle, Oxymycterus rufus, Scapteromys aquaticus, Sstaphylinid.

\section{INTRODUCCIÓN}

Las especies de la Tribu Amblyopinini (Coleoptera, Staphylinidae, Ambliopininae) son recolectadas frecuentemente del pelaje y de los nidos de roedores y marsupiales de la Región Neotropical. Sin embargo, su relación con estos mamíferos se encuentra aún en discusión ${ }^{1,2}$. Mientras algunos los consideran ectoparásitos obligados que se alimentan de la sangre de los hospedadores ${ }^{3,4}$, el estudio de especies del Género Amblyopinus Solsky sostiene que la relación de estos ambliopininos con los mamíferos es de mutualismo ${ }^{1,5}$, y que las diferentes especies se asocian a ciertas especies de mamíferos más que a otras, mostrando especificidad por el hospedador ${ }^{6}$. En Punta Lara, Argentina, la subespecie Amblyopinodes gahani gahani se recolectó de los roedores Scapteromys aquaticus y Oxymycterus rufus, pero el análisis de índices y parámetros de infestación sugieren que sería esta última especie la que garantiza la presencia de este ambliopinino en dicha localidad, siendo $S$. aquaticus un recurso alternativo ${ }^{7}$. Sobre la base de esta hipótesis, en la presente contribución se analiza la presencia de $A$. g. gahani en seis localidades ubicadas en los márgenes bonaerenses de los ríos Paraná y de la Plata en la Argentina.

\footnotetext{
* Centro de Estudios Parasitológicos y de Vectores (CEPAVE). Calle $2 \mathrm{~N}^{\circ}$ 584, 1900 La Plata, Argentina. santiagonava23@yahoo.com.ar

** CONICET, Argentina.

*** Universidad Nacional de Mar del Plata. Funes 3250, 7600 Mar del Plata, Argentina.
} 


\section{MATERIAL Y MÉTODOS}

El área de estudio se ubica en la costa de los ríos Paraná y de la Plata, en la Provincia de Buenos Aires, Argentina. Comprende un área casi rectangular paralela a la costa desde la ciudad de

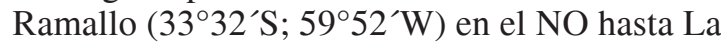
Balandra en el extremo SE $\left(34^{\circ} 56^{\prime} \mathrm{S}, 57^{\circ} 42^{\prime} \mathrm{W}\right)$. Hudson (Partido de Berazategui), Punta Lara (Partido de Ensenada), Palo Blanco y Los Talas (Partido de Berisso), constituyen localidades intermedias. El área de interés pertenece al límite sur de la selva marginal que nace en el Dominio Paranense, con características subtropicales aunque algo empobrecidas ${ }^{8}$.

Las capturas de los roedores se realizaron bajo las normas y con la autorización de la Dirección de Desarrollo Forestal y Recursos Naturales del Ministerio de la Producción de la Provincia de Buenos Aires. En Ramallo se muestreó mensualmente entre enero de 2000 y marzo de 2001; en Hudson, en abril y julio de 1995; en las restantes localidades, en abril, julio y diciembre de 1995 y 1996. En cada muestreo se utilizaron 80 trampas de captura viva y pan embebido en aceite comestible como cebo. Los roedores capturados fueron sacrificados y colocados en bolsas de polietileno para evitar el intercambio de ambliopininos. En el laboratorio, los coleópteros se obtuvieron manualmente del pelaje de los roedores bajo lupa binocular, se fijaron en alcohol $70 \%$, prepararon siguiendo las técnicas convencionales y se identificaron taxonómicamente. Ejemplares representativos de las especies de roedores y de A. g. gahani se depositaron en las colecciones de los Departamentos de Mastozoología y Entomología del Museo de la Plata (MLP), Argentina, respectivamente. A partir del registro del número de ejemplares recolectados de cada individuo roedor, se calculó la abundancia media $(\mathrm{AM}=$ número de especimenes de A. g. gahani recolectados / número de roedores de un determinado grupo taxonómico examinados) y la prevalencia $(\mathrm{P}=$ [número de ejemplares de roedores de una determinada especie asociados a A. g. gahani / número de roedores de esa misma especie examinados] X 100) $)^{9}$.

\section{RESULTADOS Y DISCUSIÓN}

Se capturaron en total 321 roedores de las siguientes especies (Muridae: Sigmodontinae): $O$. rufus $(\mathrm{N}=63), S$. aquaticus $(\mathrm{N}=157)$, Akodon azarae $(\mathrm{N}=43)$, Oligoryzomys sp. $(\mathrm{N}=47)$, Deltamys kempi, 1917)(N = 3) y Holochilus brasiliensis $(\mathrm{N}=8)$. En la Tabla 1 se observa el número y porcentaje de roedores capturados de cada especie y en cada localidad. Solamente ejemplares de $S$. aquaticus y Oligoryzomys sp. se capturaron en todas las localidades, mientras que individuos de $O$. rufus no se registraron en Ramallo ni en Palo Blanco. En esta última localidad tampoco se capturaron ejemplares de A. azarae. Las restantes especies de roedores sólo estuvieron presentes en una o dos localidades. O. rufus fue muy abundante en Hudson $(46,15 \%)$ y en Punta Lara $(40 \%)$. En oposición a esta especie, la abundancia de $S$. aquaticus varió entre el $40 \%$ y 90\% en Ramallo, Palo Blanco, Los Talas y La Balandra. Estos resultados están en relación con la preferencia por diferentes microambientes por parte de estos roedores. Mientras O. rufus selecciona ambientes húmedos pero que no se inundan, $S$ aquaticus prefiere ambientes anegados $^{10,11}$.

En total se recolectaron 181 especimenes de A. g. gahani. Setenta y siete de ejemplares de $O$. rufus y 104 de $S$. aquaticus. En ninguna de las localidades se registraron ambliopininos de $A$. azarae, Oligoryzomys sp., D. kempi y $H$. brasiliensis. Estos resultados sugieren preferencia de A. g. gahani por la especie de los roedores y son consistentes con estudios realizados en Panamá, donde de 38 posibles especies de micromamíferos, Ambyopinus emarginatus Seevers y Ambyopinus tiptoni Barrera se encontraron asociados a una sola especie hospedadora cada uno de ellos. Asimismo, experimentos realizados en el laboratorio donde a los ambliopininos se les ofreció la posibilidad de elegir entre tres especies de roedores, siempre seleccionaron aquella a la cual se encuentran asociados en ambientes naturales ${ }^{12}$.

En la Tabla 2 se muestra el número de especimenes de A. g. gahani recolectados, los valores de AM (y número máximo de especimenes) y $\mathrm{P}$ para cada especie de roedor y en cada localidad. Comparando las diferentes localidades, en Hudson, en Punta Lara y en La Balandra se recolectaron ejemplares de A. $g$. gahani tanto de $O$. rufus como de $S$. aquaticus, mientras que en Los Talas el único ejemplar capturado de $O$. rufus no estaba asociado a 
Amblyopinodes gahani gahani en localidades ribereñas de la Argentina - M. Lareschi et al.

Tabla 1. Número y porcentaje de ejemplares de cada especie de roedor capturado en cada localidad

\begin{tabular}{|c|c|c|c|c|c|c|c|c|c|c|c|c|}
\hline \multirow[b]{2}{*}{ Roedores } & \multicolumn{2}{|c|}{ Ramallo } & \multicolumn{2}{|c|}{ udsol } & \multicolumn{2}{|c|}{ Localidades } & \multirow{2}{*}{$\begin{array}{r}\text { Palo } \\
\mathbf{N}^{\circ}\end{array}$} & \multirow{2}{*}{$\begin{array}{c}\text { Blanco } \\
(\%)\end{array}$} & \multicolumn{2}{|r|}{ Talas } & \multicolumn{2}{|c|}{ La Balandra } \\
\hline & & & & & & & & & & (b) & & \\
\hline$x y n$ & 0 & 0,00 & 30 & 46,15 & 16 & 40,00 & 0 & 0,00 & 1 & 2,22 & 16 & 21,05 \\
\hline Scapteromys. aquaticus & 22 & 40,0 & 11 & 16,92 & 8 & 20,00 & 36 & 90,00 & 38 & 84,44 & 42 & 55,26 \\
\hline Oligoryzomys spp. & 8 & 14,5 & 16 & 24,62 & 9 & 22,50 & 4 & 10,00 & 2 & 4,44 & 8 & 10,53 \\
\hline Akodon. azarae & 17 & 30,9 & 7 & 10,77 & 7 & 17,50 & 0 & 0,00 & 2 & 4,44 & 10 & 13,16 \\
\hline Deltamys kempi & 0 & 0,00 & 1 & 1,54 & 0 & 0,00 & 0 & 0,00 & 4,44 & & 0 & 0,00 \\
\hline Holochilus. brasiliensis & 8 & 14,5 & 0 & 0,00 & 0 & 0,00 & 0 & 0,00 & 0 & 0,00 & 0 & 0,00 \\
\hline Total & 55 & 100 & 65 & 100 & 40 & 100 & 40 & 100 & 45 & 100 & 76 & 100 \\
\hline
\end{tabular}

Tabla 2. Número de especimenes de Amblyopinodes gahani gahani recolectados, valores de la abundancia media (AM) (y número máximo de especimenes colectados) y prevalencia $(P, \%)$

\begin{tabular}{|c|c|c|c|c|c|c|}
\hline \multirow{3}{*}{ Localidades } & \multicolumn{6}{|c|}{ Roedores } \\
\hline & \multicolumn{3}{|c|}{ Oxymycterus rufus } & \multicolumn{3}{|c|}{ Scapteromys aquaticus } \\
\hline & $\mathbf{N}^{\circ}$ & $\mathbf{A M}$ & $\mathbf{P}(\%)$ & $\mathbf{N}^{\circ}$ & $\mathbf{A M}$ & $\mathbf{P}(\%)$ \\
\hline Ramallo & 0 & - & - & 0 & - & - \\
\hline Hudson & 31 & $1,03(3)$ & 43,33 & 32 & 2,91 (9) & 63,64 \\
\hline Punta Lara & 33 & $2,06(7)$ & 62,50 & 10 & $0,75(3)$ & 75,00 \\
\hline Palo Blanco & 0 & - & - & 0 & & \\
\hline Los Talas & 0 & - & - & 7 & $0,18(4)$ & 10,53 \\
\hline La Balandra & 13 & $0,81(6)$ & 37,50 & 55 & $1,31(9)$ & 52,38 \\
\hline
\end{tabular}

ambliopininos, pero si lo estaban el $10,53 \%$ de los individuos de $S$. aquaticus. En contraposición a estos resultados, en Ramallo y en Palo Blanco, donde no se capturaron ejemplares de $O$. rufus, no se registraron ambliopininos de los ejemplares de $S$. aquaticus, cuya proporción en la comunidad fue igual o mayor al $40 \%$. Estos resultados confirman la hipótesis ${ }^{7}$ respecto de la necesidad de que $O$. rufus se encuentre en una localidad determinada para que A. g. gahani esté presente, aún asociado a otra especie hospedadora. Los resultados también en respecto de que el género Amblyopinodes Seevers se encuentra asociado primariamente a las especies de Oxymycterus, siendo la localidad típica Punta Lara ${ }^{13}$.

Sin embargo, la P de A. g. gahani fue más elevada en $S$. aquaticus que en $O$. rufus en todas las localidades. Resultados similares se obtuvieron respecto de la AM, excepto en Punta Lara en coincidencia con estudios previos realizados en la misma localidad ${ }^{7}$. Particularmente en Los Talas, donde la abundancia de $O$. rufus fue inferior al $3 \%$, los valores de AM y P de A. g. gahani asociado a $S$. aquaticus, fueron más bajos que en las restantes localidades en el mismo hospedador. Si bien el presente estudio confirma que $A$. g. gahani está restringido a determinadas especies de mamíferos, siendo $O$. rufus la especie que garantiza su presencia en una localidad determinada, surge también de los resultados obtenidos la necesidad de analizar en futuros estudios los factores que estarían influenciando la abundancia y prevalencia de este ambliopinino, información que permitirá alcanzar un conocimiento más completo de la relación de $A$. $g$. gahani con las diferentes especies hospedadoras en localidades ribereñas de la Provincia de Buenos Aires en la Argentina.

\section{RESUMEN}

Se analiza la presencia de Amblyopinodes gahani gahani (Fauvel, 1901) en localidades ribereñas de la Provincia de Buenos Aires, Argentina. De las seis especies de roedores capturados, A. g. gahani fue recolectado sólo de Oxymycterus rufus (Fisher, 1814) de Hudson, Punta Lara y La Balandra, y de Scapteromys aquaticus Thomas 1920 de las localidades mencionadas, así como de Los Talas. En las 
restantes localidades, Ramallo y Palo Blanco, donde no se capturaron ejemplares de $O$. rufus, tampoco se recolectaron especimenes de $A . g$. gahani. Los resultados obtenidos confirman conocimientos previos respecto de la relación entre este ambliopinino y $O$. rufus.

\section{REFERENCIAS}

1.- ASHE J S, TIMM R M. Probable mutualistic association between staphynilidae beetles (Amblyopinus) and their rodent hosts. J Trop Ecol 1987; 3: 177-81.

2.- TIMM R M, ASHE J S. Host and elevational specificity of parasitic beetles (Amblyopinus Solsky) (Coleoptera: Staphylnidae) in Panamá. Proc Biol Soc Wash 1987; 100: 13-20.

3.- SEEVERS C H. A new subfamily of beetles parasitic on mammals. Staphylinidae, Amblyopininae. Fld Mus Nat Hist (Zool) 1944; 28: 155-72.

4.- KIM K C. Evolutionary relationships of Parasitic Arthropods and Mammals. In: K.Ch. Kim (Ed). Coevolution of Parasitic Arthropods and Mammals: 3-82. London University Press. 1985.

5.- ASHE J S, TIMM R M. Predation by and activity patterns of «parasitic» beetles of the genus Amblyopinus (Coleoptera: Staphylinidae). J Zool Lond 1987; 212: 429- 37.

6.- TIMM R M, ASHE J S. The mystery of the Gracious Hosts. Nat Hist 1988; 9: 6-8: 25-34.

7.- LARESCHI M, CICCHINO A. Abundancia, prevalencia y preferencia de Amblyopinodes gahani gahani (Fauvel,
1901) (Coleoptera: Staphylinidae) en roedores sigmodontinos de la Reserva Selva Marginal de Punta Lara, Provincia de Buenos Aires, Argentina. Acta Zool Mex (n.s) 2002; 85: 1-9.

8.- RINGUELET R A. El ecotono faunístico subtropicalpampásico y sus cambios históricos. Symposia VI Jornadas Argentinas de Zoología: 1981; 75-80.

9.- BUSH A O, LAFFERTY K D, LOTZ J M, SHOSTAK A W. Parasitology meets ecology on its own terms: Margolis et al. Revisited. J Parasitol 1997; 83: 57583.

10.- CUETO V R, CAGNONI M, PIANTANIDA M J. Habitat use of Scapteromys tumidus (Rodentia: Cricetidae) in the delta of the Paraná River, Argentina. Mammalia 1995; 59: 25-34

11.- SÁNCHEZ LÓPEZ M I. Factores que limitan la abundancia de los roedores muridos en el Delta del Paraná. Tesis Doctoral, Universidad Nacional de Buenos Aires, Buenos Aires, Argentina. 1998. 150 pp.

12.- GETTINGER D, MARTINS-HATANO F, ESBERARD $\mathrm{C}$ et al. A Method for Testing the Host Specificity of Ectosimbionts: Give Them the Opportunity to Choose. Entomología y Vectores (inéd).

13.- MACHADO ALLISON C E. Nuevos estafilinideos parásitos de roedores y clave para las especies del Género Amblyopinodes Seevers, 1955. (Col. Staphylinidae). Papéis Avulsos. Dept Zoologia São Paulo Brasil 1962; 15: 81-90.

Agradecimientos: A Ulyses F.J. Pardiñas (CENPAT, Argentina) y Carlos Galliari (Zoológico de La Plata, Argentina) por la determinación de los roedores. 